\title{
Systematic Review: de Novo Dynamic Spine Stabilization
}

\section{Eyad Faizo MD*}

Murnau hospital, Murnau, Germany

Submission: November 16, 2017; Published: January 19, 2018

*Corresponding author: Eyad Faizo, MD, Neurosurgery Resident, Murnau hospital, Murnau , 82418 Germany, Tel: 00491742043838; Email: Dr.Faizo@gmail.com

\section{Abstract}

Intervertebral disc (IVD) degeneration is considered one of the main causes of pain and extreme life quality retrogradation. Previously, the main alternative for medical pain control was the surgical adjustment of the spine through fusion of the vertebrae, through elimination of the degenerative plates, while adding a bone graft material and anchoring by a device to hold everything together. Posterior dynamic stabilization of the lumbar spine had experienced a noteworthy advancement in the most recent decade. In spite of the expanding utilization of this system, few prospective studies have been directed to evaluate its outcomes and convenience. In our review, we are surveying the literature for prospectively designed studies evaluating different techniques and devices of posterior dynamic lumbar stabilization.

Keywords: Spine; Spinal stenosis; Dynamic; Posterior; Lumbar

\section{Introduction}

Intervertebral disc (IVD) degeneration is considered one of the main causes of pain and extreme life quality retrogradation. Previously, the main alternative for medical pain control was the surgical adjustment of the spine through fusion of the vertebrae. This surgical technique involves elimination of the degenerative plates, while adding a bone graft material and anchoring by a device to hold everything together. The real issues with this principle are the extra weight on the vertebra above and beneath the combination point, and the rigid fixation and limitation of movement.

Posterior dynamic stabilization of the lumbar spine had experienced a noteworthy advancement in the most recent decade. Dynamic adjustment devices assure to mitigate difficult spinal development by changing the transmission of body loads over degenerative plates and avoiding the negative outcome of pressure over it .The mechanism of such gadgets work through confining spine movement over the sored plate and applying differing degrees of movement control at different anatomical parts of impact which prevents progressive worsening of the condition while providing natural controlled motion of the spine.

Clinical reports over the posterior dynamic stabilizations proved that it is a quickly growing field with a wide range of devices, methodologies, and surgical approaches. In spite of the expanding utilization of this system, few prospective studies have been directed to evaluate its outcomes and convenience. In our review, we are surveying the literature for prospectively designed studies evaluating different techniques and devices of posterior dynamic lumbar stabilization.

\section{Methods}

A systematic search was conducted through Medline database with the aim to identify as many studies on the topic of posterior dynamic lumbar spine stabilization. A comprehensive search was applied utilizing identified search terms including posterior, dynamic, lumbar spine, stabilization, and commencement. The inclusion criteria for article selection were: Prospective clinical trial utilizing at least one technique of dynamic lumbar stabilization for patients with a degenerative lumbar disease. The exclusion criteria were: Articles written in a language other than English. A follow up less than 12 months or Articles published before January 2014. Search were performed by three autonomous neurosurgeons.

The abstracts were reviewed to assure their relevance to the subject of the research and if no exclusion criteria were distinguished, the full manuscript was therefore acquired. Appraisals of methodological quality were made by AMSTAR and PRISMA were applied to all meta-analyses found in our search. A total of 85 articles were searched, of which 78 were excluded. The study was based on the remaining 7 articles. 
Results of different papers were analysed and outcomes were classified according to the incidence of complications or the efficacy of the surgery and the achieved physical activities.

\section{Results}

Seven publications were included in our analysis. Devices included in our review are: Dynesys, PercuDyn, Wallis, Wallis, Isobar. In 2015, Haodong Fei et al. [1] reported a comparison between radiographic and clinical outcomes of posterior dynamic stabilization(PDS) versus posterior lumbar intervertebral fusion (PLIF) in treating degenerative disc disease (DDD). They prospectively designed a study for a period of 3 years and successfully consented patients were divided into two groups either for having Dynesys implantation or the standard lumbar intervertebral fusion. The Dynesys dynamic adjustment system is a contrasting option to inflexible instrumentation for the treatment of lumbar degenerative infection. Their result showed a lesser intraoperative blood loss and drained volume for the PDS group. Similarly, the PDS group did have a shorter hospital stay and a decrease in total expenses of surgery in comparison to the Fusion group. There were no significant differences across both groups regarding back visual analogue scale (VAS), leg VAS, Oswestry disability index (ODI) scores and incidence of complications.

Careno et al. [2] reported treatment of 96 patients with lumbar discogenic pain associated with facet pain and canal stenosis surgically treated with the PercuDyn device. Both VAS scale and the ODI score showed a significant difference at 1,6-month, and 2-year follow-up with respect to the preoperatory. The drawbacks taking on such study was avoiding using a control group. Only (14.3\%) received revision surgery with posteriorstatic stabilization using bars and screws because of mobilization of the device with worsening of symptoms.

Yuan X et al. [3] reported utilization of Wallis interspinous dynamic fixation for after posterior lumbar decompression in patients with L4-5monosegmental lumbar disc herniation. The result revealed that VAS (back pain), VAS (leg pain) and ODI showed significant improvement at the end of the follow up. At the final follow-up, IDH of the upper and lower adjacent segment showed no significant difference compared with those preoperatively $(\mathrm{P}>0.05)$; ROM of the upper adjacent segment increased $(\mathrm{P}<0.05)$, while ROM of the lower adjacent segment did not change significantly compared with that preoperatively $(\mathrm{P}>0.05)$. The degeneration of intervertebral discs at the upper and lower segments showed no significant changes at the final follow-up ( $\mathrm{P}>0.05)$.

Daentzer et al. [4] utilized Wallis spacer fort en patients with therapy resistant or progressive back and/or leg pain under conservative treatment due to spinal canal stenosis and were controlled clinically and radiologically after 3, 6, 12, and 24 months Results showed that back pain intensity was significantly reduced with a mean value of 6.0 on visual analog scale (VAS) before surgery and of 2.7 at the latest evaluation. Meantime leg pain intensity was reduced but without statistically significant difference. The functional disability according to Oswestry Disability Index (ODI), Roland-Morris Disability Questionnaire (RM) and the health related quality of life were reduced with statistically significant difference.

Barbagallo GM \& Certo F [5], employment of a screws, hybrid rods (Isobar ${ }^{\mathrm{TM}}$ Evolution, Scient'X Alphatec Spine) as well as interbody cages for treatment of three patients suffering from degenerative spondylolistesis and adjacent level's degenerative disc disease. Their results have shown prominent decrease in the mean Visual Analogue Scale (VAS) scale and the Oswestry Disability Index (ODI) questionnaire score, from 8.3, preoperatively, to 5 and from 72.66 to 43.98 , respectively.

Oktenoglu et al. [6] established a prospective study comparing the clinical results ofanterior lumbar total disc replacement versus the posterior transpedicular dynamic stabilization in the treatment of degenerative disc disease. The study was conducted between 2004 and 2010 with a total of 50 patients. Their results showed that posterior transpedicular dynamic stabilization have a significantly lower intraoperative blood loss and operative time $(\mathrm{p}<0.01)$. Regarding preoperative VAS and ODI levels both groups did show improvement with no significant differences.

Krappel $\mathrm{F}$ et al. [7] in a prospectively designed study randomised 146 patients with a single level disc herniation (L2 to L5) as: 75 investigational (herniectomy and DIAM) and 71 control (herniectomy alone) treated and followed up for 24 months. Their result confirmed significant improvement for both groups without a significant difference between both groups at 6 and 24 months.

\section{Discussion}

Lumbar spinal stenosis (LSS) is a degenerative disc disease resulting in narrowing of the spinal channel, and combined with agonizing low back pain and sciatica, which is exacerbated with walking. The standard first line of care would be conservative in utilizing analgesics, NSAIDs, and epidural steroid infusion. Patients showing signs of failure of the conservative management undergo laminectomy for decompression of the nerve roots. Fusion have been considered the golden treatment for difficult degenerative states of the lumbar spine that have been resistant to non-operative treatment. For quite a while, decent outcomes were thought to be reliant radiological postoperative assessment.

Notwithstanding different reports have challenged this success with the impaired range of motion resulting from fusion surgery. In the meantime, the results of pain alleviation have been sub satisfactory in a large category of patients. In this manner, a hypothesis has been generated and widely accepted that surmised a reduction of segmental motion rather than 
fusion and fixation would be more appropriate for elimination of pain and preservation of partial spine movements. Posterior dynamic stabilization of the lumbar spine have empowered the treatment of spinal agony and lumbar degenerative diseases without undesired limitations of the spinal movement associated with the gold standard fusion surgery.

Based on that hypothesis, Haodong Fei et al. [1] reported in a prospective cohort study that the short and long term functional outcomes demonstrate similar outcomes between posterior dynamic stabilization(PDS) versus posterior lumbar intervertebral fusion (PLIF)on leg and back VAS or ODI scores. However, PDS have showed significant advantages on blood loss, length of stay in hospital, total charge, and radiographic outcomes.

Posterior dynamic stabilization was responsible for the popularity of the interspinous gadgets market (X STOP, Wallis, DIAM, ExtenSure, Coflex, Aperius PercLID) that proved to provide a less meddling alternative for spinal fixation surgery in order to increase the neural foramen. These interspinous inserts are usually made of one piece comprised of either titanium, or other materials such as polyether ether ketone [PEEK].

Careno et al. [2] reported a more than 70\% patient's satisfaction with the PercuDyn device with a considerably long follow up period reaching to 2 years, but with a major study limitation represented in absence of randomization or a control group. A Yuan X et al, report regarding Wallis interspinous dynamic fixation over a 5 years follow up period achieved a satisfactory clinical results. Similarly, Daentzer et al. [4] reported significant improvement of the iROM of the treated segments after operation and after 3 and 12 months but without a significant decreased in ROM after 6 and 24 months when compared to the preoperative value.

Isobar have been deployed by Barbagallo GM \& Certo F [5] reported, showing a mean VAS and ODI score reduced from 8.3, preoperatively, to 5 and from 72.66 to 43.98 , respectively without any reported complications. Oktenoglu et al. [6] compared between total disc replacement (TDR) and posterior transpedicular dynamic systems (PTDS) and their results were similar to Haodong Fei et al. [1] favouring dynamic stabilization as a procedure with less intraoperative blood loss and operative time.

Krappel F et al. [7] compared herniectomy alone versus DIAM and there was no significant difference across both groups outcomes. In view of our audit of the available peer-reviewed articles, we conclude that interspinous dynamic stabilization is a reliable and efficient treatment.

However, the technique has its limitations which we summarize as follows: (1) its theoretical background has not been established, (2) its indications and available study results are inconsistent, and (3) the results of long-term follow-ups and randomized clinical studies that compare it with other treatment methods are inadequate [8-12].

Thus, because various conventional treatment methods are currently available for target diseases, we conclude that more concrete evidence of the safety and efficacy of interspinous dynamic stabilization is required. A well-designed study should be undertaken to provide more concrete evidence of its merits.

\section{Referances}

1. Fei H, Xu J, Wang S, Xie Y, Ji F, et al. (2015) Comparison between posterior dynamic stabilization and posterior lumbar interbody fusion in the treatment of degenerative disc disease: a prospective cohort study. J Orthop Surg Res 10:87.

2. Canero G, Carbone S (2015) The results of a consecutive series of dynamic posterior stabilizations using the PercuDyn device. Eur Spine J Suppl 7: 865-871.

3. Yuan X, Kong C, Chen XM, Cui LB, Xu SJ, et al. (2016) The mid- to longterm effect of Wallis lumbar interspinous dynamic stabilization on the adjacent segment degeneration. Zhonghua Wai Ke Za Zhi 54(12): 914918.

4. Daentzer D, Hurschler C, Seehaus F, Noll C, Schwarze M (2016) Posterior dynamic stabilization in the lumbar spine-24 months results of a prospective clinical and radiological study with an interspinous distraction device. BMC Musculoskelet Disord 17:90.

5. Barbagallo GM, Certo F (2017) The unfeasible made feasible: lumbar minimally invasive hybrid stabilization with dynamic rod and miniopen TLIF. J Neurosurg Sci

6. Oktenoglu T, Ozer AF, Sasani M, Ataker Y, Gomleksiz C, et al. (2013) Posterior transpedicular dynamic stabilization versus total disc replacement in the treatment of lumbar painful degenerative disc disease: a comparison of clinical results. Adv Orthop 2013: 874090.

7. Krappel F, Brayda-Bruno M, Alessi G, Remacle JM, Lopez LA, et al. (2016) Herniectomy versus herniectomy with the DIAM spinalstabilization system in patients with sciatica and concomitant low back pain results of a prospective randomized controlled multicenter trial. Eur Spine J.

8. Bonaldi G, Brembilla C, Cianfoni A (2015) Minimally-invasive posterior lumbar stabilization for degenerative low back painand sciatica. A review. Eur J Radiol 84(5): 789-798.

9. Ham MH, Mehta VA, Patel NN, Jakoi AM, Hsieh PC, et al. (2016) Complications associated with the Dynesys dynamic stabilization system: a comprehensive review of the literature. Neurosurg Focus 40(1): E2.

10. Kaner T, Ozer AF (2013) Dynamic stabilization for challenging lumbar degenerative diseases of the spine: a review of the literature. Adv Orthop 2013: 753470.

11. Shea BJ, Hamel C, Wells GA, Bouter LM, Kristjansson E, et al. (2009) AMSTAR is a reliable and valid measurement tool to assess the methodological quality of systematic reviews. J Clin Epidemiol 62(10): 1013-1020.

12. Moher D, Liberati A, Tetzlaff J, Altman DG (2009) Preferred reporting items for systematic reviews and meta-analyses: the PRISMA statement. PLOS Med 6: e1000097. 
Your next submission with Juniper Publishers will reach you the below assets

- Quality Editorial service

- Swift Peer Review

- Reprints availability

- E-prints Service

- Manuscript Podcast for convenient understanding

- Global attainment for your research

- Manuscript accessibility in different formats

( Pdf, E-pub, Full Text, Audio)

- Unceasing customer service

Track the below URL for one-step submission https://juniperpublishers.com/online-submission.php 